ISNN 1810-5491

Instituto de Estudios Nacionales

Cuadernos Nacionales

$\mathrm{N}^{\circ} 28$, enero -junio, 2021

\title{
LA PARTICIPACIÓN COMUNITARIA EN EL PROCESO DE DESCENTRALIZACIÓN DEL MUNICIPIO DE SAN MIGUELITO: LOGROS Y LIMITACIONES.
}

\author{
Milka Ruiz de Galástica ${ }^{1}$ \\ Universidad de Panamá \\ milkaicela@hotmail.com
}

\section{Resumen}

Este artículo nos inserta en el proceso de descentralización aprobado legalmente en el país, que deja de lado la práctica del centralismo para dar paso a la inclusión de la población en la toma de decisiones de las acciones que solventarán las necesidades y problemas sociales en procura de un mayor desarrollo local. Para este análisis se utilizó la revisión documental de fuentes bibliográficas para focalizar algunas teorías que sustentan la práctica de la participación, y la consulta a las normativas que plasman la concepción del proceso de descentralización concluyendo que el componente de la participación ciudadana es vital para el desarrollo de la descentralización, pero que aún existen prácticas discursivas no coherentes con la participación comunitaria.

PALABRAS CLAVES: descentralización, participación, participación comunitaria, gestión local, desarrollo local.

\begin{abstract}
This article inserts us into the decentralization process legally approved in the country, which sets aside the practice of centralism to give way to the inclusion of the population in decision-making of actions that will solve social needs and problems in search of greater local development. For this analysis, the documentary review of bibliographic sources was used to focus on some theories that support the practice of participation, and the consultation of the regulations that embody the conception of the decentralization process, concluding that the component of citizen participation is vital for development. decentralization, but there are still discursive practices that are not consistent with community participation.
\end{abstract}

1. Centro Regional Universitario de San Miguelito. 
KEYWORDS: decentralization, participation, community participation, local management, local development.

\section{INTRODUCCIÓN}

En todo proceso de descentralización, es decisivo aplicar mecanismos de participación comunitaria para dinamizar el componente democrático en la eficacia del proceso, convirtiendo a los municipios en espacios geo-políticos favorables en el desarrollo local, con la acciones y toma de decisiones de los actores sociales que lo integran.

Este artículo analiza la participación comunitaria como un elemento fundamental en el proceso de descentralización implementado en el país, particularmente en el Municipio de San Miguelito. Aborda la relación de los conceptos de participación y descentralización en los gobiernos locales para satisfacer las demandas de la población con la intervención directa de ésta en la decisión y respuestas eficaces.

\section{DESARROLLO}

\section{¿Qué es Participación Comunitaria?}

El concepto de participación es utilizado para referirse a distintos significados, muchas veces ambiguo o con posturas políticas intrínsecas, dependiendo de quien o quiénes se ocupan de ella. Aunque resulta ser un término de uso cotidiano, la Real Academia de la Lengua Española (2017) indica que la palabra participación "procede del latín participare, y está compuesta de la raíz pars (parte) y del derivado capere (tomar, agarrar), lo que significa tomar parte en algo, recibir una parte de algo, o compartir algo como acción y el efecto de hacer participe, repartir", pero aún denota su amplitud e imprecisión.

Lo que pareciera interesante de esta definición es que conlleva a concretar que una persona logra algo, siempre y cuando está en correspondencia o relación de o con otros, por lo tanto, el carácter individualista no existe, siendo una relación de mutua transformación, donde el participante construye y modifica al objeto o hecho en el cual participa, y por el hecho de hacerlo, es también transformador (Montero, 2006). 
Hoy día, uno de los usos otorgados al término de participación por los Estados y organismos nacionales e internacionales, está vinculado a los acontecimientos de los individuos y grupos sociales en los asuntos de interés público, dándose un renombre que muchas veces, solo queda retórico cuando se utiliza como simple instrumento para el logro de un interés particular. Carmona (2003) señala que la participación debe plantearse como un derecho a través del cual se puede lograr la autorrealización, cuando permite tomar decisiones y acciones conjuntas, y a su vez, establece un cambio de relación, refiriéndose al equilibrio de fuerzas sociales y al poder. Para Burin, Karl y Levin (1998) constituye un proceso social, que supone un ejercicio permanente de derechos y responsabilidades.

Estos planteamientos enmarcan la participación como la construcción del derecho de todos los individuos, capaz de incidir en las formas de dominio y autoridad existentes, pero también, implica que se forja de manera consciente y voluntaria (Harnecker y Bonilla-Molina, 2005), sin imposiciones para el logro del bien común y satisfacer una necesidad colectiva. Siendo así, se refiere al hecho que todo aquel que participa reconoce su derecho y de su accionar para lograr los propósitos. Desde la clasificación realizada por las Naciones Unidas (1976) en el Pacto Internacional, es ubicada dentro de los derechos civiles y políticos que protegen las libertades individuales y garantizan que cualquier ciudadano pueda participar en la vida social y política en condición de igualdad y sin discriminación, con libertad de opinión y de expresión, de reunión pacífica, de libertad de asociación, y la participación en asuntos públicos y elecciones.

Como se indicó al inicio, la participación es un vocablo con muchos usos a la cual se coloca un calificativo para precisar el tipo de participación de que se trata, por ejemplo: política, comunitaria, ciudadana, ambiental, entre otras. Para Sánchez y Del Pino (2008), existe equivalencia en los términos participación ciudadana, comunitaria y social, y Cunill (2004) hace la salvedad que la participación ciudadana comprende todas aquellas experiencias que remiten a la intervención de los individuos en actividades públicas para hacer valer intereses sociales. A nuestro modo de ver, la participación comunitaria va más allá de otras participaciones colectivas porque alude al mismo tiempo el concepto de comunidad, que implica entre otros elementos los 
límites de demarcación, y el sentido de pertenencia de los individuos (Orozco, 2018) en el cual se llevan a cabo acciones pensando en el bien colectivo y sus compromisos y responsabilidades co-existen para y por las satisfacciones de todos, y por lo tanto, podemos decir que, es un sistema de relaciones sociales permanentes, integrada en base a intereses y necesidades sentidas y compartidas, donde los miembros se preocupan unos por los otros, desarrollando las capacidades de trabajo grupal, de liderazgo y propicia la organización social como plataforma en el logro de que sus demandas serán atendidas. Desde esta perspectiva, la referencia a la comunidad nos ubica en el espacio geográfico micro social en un nivel que supera el sentido de intereses particulares y, por lo tanto, involucra a la población local en la reflexión de sus problemas, necesidades o conflictos comunes, aumentando la responsabilidad del ciudadano como sujeto. (Sánchez, Tovar y Del Pino, Espejo, 2008)

\section{El Proceso de descentralización como una alternativa transformadora en la democratización.}

En el estudio realizado por Finot (2002) respaldado por la CEPAL acerca de la Descentralización en América Latina, se muestra que la descentralización sería el medio por excelencia para que el Estado desempeñara las nuevas funciones que le corresponden en forma eficiente, permitiendo además una mayor eficiencia fiscal: (i) una mejor adecuación de la provisión de servicios públicos a las diferencias territoriales, (ii) la descongestión de la administración pública y (iii) una mayor participación ciudadana en los procesos de asignación y control del gasto.

Maldonado (2002) refiriéndose a la descentralización política sostiene que la misma abarca la entrega de poder a dos actores sociales: a los ciudadanos para que elijan a sus propios gobernantes, y a las autoridades locales para que tomen decisiones autónomas sobre el diseño e implementación de las políticas de desarrollo local.

Por su parte, el Programa de las Naciones Unidas para el Desarrollo expresa la necesidad de "desarrollar capacidades a la población, organizaciones y sociedades que fortalezcan y mantengan las aptitudes necesarias para establecer y alcanzar sus propios objetivos de desarrollo" (PNUD, 2009:3). Esto plantea las transformaciones positivas en el empoderamiento a las 
personas, líderes y grupos organizados con competencias idóneas para desarrollar una participación comunitaria generada y sustentada de forma endógena a lo largo del tiempo, para el logro de un cambio de pensar y de actuar basado en las experiencias y saberes compartidos de quienes participan.

Como se puede apreciar, la existencia de la participación es ineludible, es el denominador común en los planteamientos esbozados de la descentralización de la población, pero también, hay que subrayar la necesidad de capacitar a estos colectivos para adquirir el carácter consciente, responsable, dinámico y organizativo que conlleva la participación, y tomando en consideración las propias particularidades de las poblaciones asentadas en cada espacio geográfico lo que obliga a los planificadores a diversificar los programas de este tipo de educación en los diferentes escenarios donde se está implementando la descentralización.

Como lo explica Finot (2002), la descentralización se define como un proceso de trasferencia organizada y ordenada del gobierno nacional a otra autoridad local, con atribuciones gerenciales y capacidades para administrar recursos humanos, económicos y financieros regionales, departamentales o municipales, con el fin de mejorar la eficiencia del Estado en cuanto a la redistribución social, con programas que enfrenten la pobreza, aumenten la participación ciudadana y reduzcan la corrupción; en definitiva, tiene el propósito de lograr mayor gobernabilidad democrática.

Desde esta mirada, emprender una descentralización obliga a los gobiernos locales a establecer alianzas entre los diferentes actores sociales y demás ámbitos gubernamentales para gestionar y demandar los recursos para la satisfacción de las demandas locales. Siendo así, la descentralización contribuye a mejorar la planificación local, en la toma de decisiones de las necesidades reales del colectivo, en la corresponsabilidad dirigida a apoyar las disposiciones de los gobiernos en programas y proyectos encaminados a mejorar la calidad de vida, y en la elaboración de propuestas objetivas y de beneficio a la comunidad. En consecuencia, es necesario que los gobiernos estatales, municipales y locales brinden a la descentralización el peso que tiene en la planeación y diseño de políticas públicas locales con acciones coordinadas y efectivas. 


\section{La participación de los ciudadanos en la normativa panameña del proceso de descentralización.}

En Panamá, el proceso de descentralización está fundamentado en la Ley 66 de 29 de octubre de 2015, que reforma la Ley 37 de 2009 que descentraliza la Administración Pública y plantea que es el "proceso gradual mediante el cual el Órgano Ejecutivo traslada competencias y responsabilidades, transfiriendo...los recursos para la implementación de políticas públicas provinciales, comarcales y municipales...en el marco de una activa participación de los ciudadanos..." (Ley No 27901-A, 2015).

La ley indica la posibilidad de lograr la eficacia del proceso del traslado de competencias y recursos, siempre y cuando se consolide la participación de la población, pues ésta es su garantía. Y agrega, que, "la participación ciudadana es la acción consciente, deliberada, participativa, inclusiva y organizada de la comunidad...” (Ley No 27901-A, 2015, art. 136)

De esta manera, son destacados algunos componentes propios de la participación comunitaria, los cuales terminan siendo fundamentales para distinguir el concepto de participación, como lo es, la existiencia de una determinación voluntaria de los ciudadadanos a ser "parte de", sin embargo, la población aún no visualiza este derecho democrático, al igual que la responsabilidad que le corresponde en la toma de decisiones de las políticas públicas como una forma distinta de incidir en éstas desde los ámbitos locales. La ley incorpora la organización de la población en la participación, proporcionando un carácter de articulación y estructura asociativa requerida para que la gente se integre, forme parte y actúe colectivamente, por consiguiente, es contraria a la espontaneidad y naturalidad, hay que promoverla y propiciarla a través de mecanismos donde las personas pasen de ser simples espectadores a actores, a no solo recibir o ejecutar acciones, sino tomar decisiones, en otras palabras no se convierta en concepto retórico para responder a intereses particulares.

Montecinos (2005) sustenta que la descentralización es una actividad colectiva en donde los actores sociales gozan de cierta capacidad o poder de influencia en las decisiones que les conciernen. Se parte del supuesto de que la vida local constituye el medio más natural para 
fortalecerla y por consiguiente la participación comunitaria es la que impregnará movilidad a las acciones pretendidas en la aplicación de la ley de descentralización.

También hay que analizar los niveles de participación de los colectivos sociales. Es muy común encontrar proyectos comunitarios donde las opciones fundamentales se deciden entre especialistas o tomadores de decisiones exógenos y sólo se consultan a las "bases" para aspectos operativos de ejecución o de logística relativamente secundarios. Esta irregularidad de la participación comunitaria es apreciada hasta en las organizaciones que hacen alarde de su beligerancia en la misma, y cuando se aborda el tema de los recursos financieros apelando al control y confidencialidad de éstos.

En efecto, es imposible hablar de participación mientras no exista un control y plena transparencia sobre los recursos del presupuesto municipal para una auténtica democracia participativa y el empoderamiento local.

\section{El Municipio de San Miguelito en la construcción del modelo descentralizador de gestión local.}

La cultura de participación ciudadana en el país ha tenido altas y bajas. En los 115 años de fundación de vida republicana, se han tejido hechos históricos que marcan la democracia panameña.

Fueron impulsados, durante el gobierno militar de 1968, algunos acontecimientos de participación social. Entre ellos, las organizaciones de los asentamientos campesinos, a quienes el gobierno les otorga tierras para su producción, expropiándolas a los terratenientes, las organizaciones sindicales y cooperativas igualmente fueron bastiones en distintos ámbitos, particularmente políticos y gremialistas, pero con la vuelta al poder de la clase oligárquica en el 98, estos grupos son reducidos.

El Distrito de San Miguelito posee una vasta historia e incidencia en la participación comunitaria. Constituyó el escenario geográfico que acogió la migración masiva de la población de escasos recursos proveniente de diferentes regiones del país, y donde se gestan las luchas colectivas para la obtención de tierras para viviendas y la atención de las demandas sociales. Valga agregar que fue ejemplo en la conformación de las estructuras organizativas populares del país y el referente 
para el establecimiento de la Asamblea Nacional de Representantes de Corregimiento, como parte del órgano legislativo (Ley $\mathrm{N}^{\circ} 17210,1972$ ), conocida actualmente como Asamblea Nacional.

Esta breve descripción de los aportes del Distrito de San Miguelito a la participación comunitaria pudiera pensarse que facilita la implementación del proceso de descentralización; no obstante, se aprecian señales que perpetúan el paternalismo cuando los ciudadanos permanecen en espera de lo que ofrecen algunas autoridades.

En el año 1999 se crea la Coordinación para la Descentralización y el Desarrollo Local (CONADEL). Esta coordinación de organismos de la sociedad civil logró que los candidatos presidenciales firmaran el Pacto por la descentralización y el desarrollo local en la que fue definida como "un proceso de transferencia gradual, equitativa y responsable de competencias, recursos humanos, tecnológicos y económicos, responsabilidades y poder de decisión, desde el gobierno central hacia los gobiernos locales.”. (CONADEL, 1999, p.3).

En el año 2004 se emprende otro paso en el camino de la descentralización al reformarse el capítulo 2 del título VIII de la Constitución Política de la República de Panamá. En esta reforma se ratifica el carácter autónomo del municipio y se delimitan las competencias propias del mismo. El artículo 233 de la Carta Magna se refiere a los municipios como entidad principal de la división político-administrativa del Estado, con gobierno propio, democrático y autónomo, y al que le corresponde prestar los servicios públicos, construir las obras públicas, ordenar el desarrollo de su territorio promover la participación ciudadana, así como el mejoramiento social y cultural de sus habitantes. (Ley $\mathrm{N}^{\circ} 25176,2004$ )

Históricamente el carácter centralista del poder del Estado ha puesto en segundo plano el valor de la participación comunitaria por lo que, las dudas sobre el grado de viabilidad en el proceso de descentralización parecieran cobrar fuerza debido al sostenimiento de una cultura con prácticas paternalistas, persistiendo la pasividad de la población y la poca consistencia de los espacios públicos democráticos para el logro de una participación comunitaria efectiva. 


\section{Logros y limitaciones de la participación comunitaria en el proceso de descentralización.}

La Ley de descentralización en Panamá establece un listado por áreas para destinar los recursos y señala a los alcaldes y representantes de corregimientos como figuras que identificarán las necesidades de las comunidades con la participación de éstas, ejecutando las obras y proyectos con los que darán respuestas. Estas autoridades, aunque elegidas por voto popular, siguen siendo dirigidas desde las estructuras de poder, lo que pudiera inducir y manejar las decisiones del colectivo poblacional o buscar nuevas formas disociadas de las necesidades reales y sus respuestas efectivas.

En el Boletín digital "Descentralización Avanza" (2016) la Secretaría Nacional de Descentralización comunica al país las actividades realizadas, resaltando que para esa fecha se ejecutaron 1,369 proyectos con un monto total aprobado de 306, millones de dólares. En el año 2018, este boletín muestra datos parciales para el cumplimiento de la descentralización, focalizando sus resultados al total de 616 millones de dólares destinados por el gobierno central a los 77 municipios en el país para 16,101 proyectos, desde la puesta en marcha del proceso. Los datos reflejan el aumento significativo en materia de las inversiones económicas para los proyectos, no obstante, las informaciones con respecto al impacto de la participación social no son presentadas, indicando tan solo, que se han realizado 2 mil procesos de participación, sin abordar la temática del tipo de participación desarrollada y los cambios sustanciales de empoderamiento y toma de conciencia de las bondades admitidas en la descentralización.

Esto nos lleva a pensar que, el proceso tal como es planteado puede conllevar a perpetuar las mismas prácticas de los poderes hegemónicos existentes en los gobiernos centralistas y a reproducir los mismos, en condiciones de un escenario o contexto político social local. Esto se sustenta en que toda administración existente, tiende a separarse con respecto de los administrados y a reducirlos en simples objetos de procedimientos regulados abstractamente (Adorno, 1997). Desde la Teórica Crítica, el proceso de descentralización se convierte en otra representación más de dominio de la cúpula central y aquellos que dirigen los cargos administrativos de las entidades gubernamentales, buscarán perpetuar nuevas formas de 
dominación de los ciudadanos; y, como señala Monje-Reyes (2015) logran su objetivo de sometimiento.

\section{CONCLUSIONES}

- La participación comunitaria es un derecho de todos los individuos, siendo capaz de incidir en las formas de dominio y autoridad existentes, pero también se forja de manera consciente y voluntaria a través de la cual la gente, las organizaciones y comunidades logran las competencias para el empoderamiento en la toma de decisiones.

- La descentralización como un proceso de trasferencia organizada y ordenada del gobierno nacional a otra autoridad o institución local, brinda capacidades a la población con atribuciones gerenciales y competencias para administrar recursos con la aprobación de programas que aumenten la participación ciudadana y reduzcan la corrupción; en definitiva, tiene el propósito de lograr mayor gobernabilidad democrática.

- El proceso de descentralización puede perpetuar las mismas prácticas de los poderes hegemónicos existentes en los gobiernos centralistas y a reproducir los mismos en condiciones de un escenario o contexto político social local, si es aplicado a la población local como simple medio de procedimiento regulado.

- El Municipio de San Miguelito debe apostar a que sus arraigos de una cultura de participación comunitaria, vuelvan a potenciarse en la población actual, con nuevos protagonistas, pero con el sostenimiento de las luchas organizadas para atender las necesidades y conflictos sociales que la realidad de hoy les muestra y desafía.

- Los estamentos institucionales constituyen un componente fundamental para propiciar el proceso descentralizador, manteniendo la claridad de las metodologías y estrategias de la participación comunitaria como proceso organizativo, colectivo, voluntario, incluyente, en el cual hay una variedad de actores, de actividades y de grados de compromiso. 
- El ámbito o espacio de aprendizaje de la participación es el propio territorio donde reside la población, donde la dimensión relacional es fortalecida, pero a su vez, son importantes los mecanismos de participación para incidir en la interacción con los demás, en el que fluya la tolerancia, la mediación y consenso para el logro de bienes colectivos.

\section{REFERENCIAS}

Adorno, W. (1997). Epistemología y Ciencias Sociales. (http://www.afoiceeomartelo.com.br/posfsa/autores/Adorno,\%20Theodor/ADORNO,\%20Theodo r\%20-\%20Epistemologia\%20y\%20ciencias\%20sociales.PDF).

Bernaza, C. (2004). Acerca de la Participación Ciudadana y el Protagonismo Social. Argentina: Instituto Provincial de la Administración Pública. (http://biblioteca.municipios.unq.edu.ar/modules/mislibros/archivos/p_c.pdf).

Burin, D., Karl, I., Levin,L. (1998). Hacia una participación efectiva y eficaz. Argentina: ediciones CICCUS.

Carmona Tinoco, J. (2003). “Algunos aspectos de la participación de México ante los órganos del sistema interamericano de protección de los derechos humanos". Cuestiones Constitucionales, (9),3-54 ISSN: 1405-9193. (Disponible en https://www.redalyc.org/articulo.oa?id=885/88500901)

CONADEL. (1999). "Coordinación para la descentralización y el desarrollo local Pacto por la descentralización y el desarrollo local”. Panamá.

Cunill, N. (2004). "Balance de la participación ciudadana en las políticas sociales. Propuesta de un marco analítico”. (pág. 56-76) en Participación ciudadana y políticas sociales del ámbito local,

Ziccardi, A. (Coordinadora) INDESOL, México.

(http://ru.iis.sociales.unam.mx/jspui/bitstream/IIS/4420/1/Participacion\%20ciudadana\%20y\%20p oliticas\%20sociales\%20en\%20el\%20ambito\%20local.pdf)

Finot, I. (2002). "Descentralización en América Latina: Teoría y práctica". (UN.CEPAL. ILPES, serie Gestión Pública No 12131 págs. Santiago de Chile. (https://repositorio.cepal.org/bitstream/handle/11362/7261/1/S01030319_es.pdf) 
Harnecker, M., Bonilla, L. (2008). "La descentralización ¿fortalece o debilita el estado

Nacional?" Centro Internacional Miranda. (http://www.spentamexico.org/v4-

n1/4\%281\%29\%20179-193.pdf).

Maldonado, A (2002). “Avances y resultados de la descentralización política en Colombia". En Departamento Nacional de Planeación. Evaluación de la Descentralización en Colombia: balance de una década. Tomo II. Análisis y resultados sectoriales, Bogotá D.C., p. 266.

Monje-Reyes, P. A. (2015). "La Teoría Crítica como perspectiva de análisis de las relaciones de dominación y los procesos de descentralización en Chile". Cuadernos EBAPE.BR, 13(2), 402409. https://doi.org/10.1590/1679-395116756.

Montecinos, E. (2002). "Planificación, participación y territorio: el caso del hospital de Río Bueno", Chile. Ther, F. (comp.) Antropología y estudios regionales. De la aplicación a la acción. Universidad de Los Lagos: Ediciones CEDER.

Montero, M. (2006). "Hacer para transformar: el método en la psicología comunitaria". Buenos Aires: Paidós. (https://www.academia.edu/30976819/Hacer_Transformar_M_Montero).

ONU. (1976). "Pacto Internacional de Derechos Civiles y Políticos". Ciudad de

Nueva York, Estados Unidos. (https://www.ohchr.org/sp/professionalinterest/pages/ccpr.aspx).

Huerta Orozco, A. (2018). "El sentido de pertenencia y la identidad como determinante de la conducta, una perspectiva desde el pensamiento complejo". IE Revista de investigación educativa $\begin{array}{llll}\text { de la REDIECH, } 9 & \text { 83-97. }\end{array}$ (http://www.scielo.org.mx/scielo.php?script=sci_arttext\&pid=S2448$85502018000100083 \& \operatorname{lng}=\mathrm{es} \&$ tlng=es).

PNUD. (2009). Desarrollo de Capacidades: Texto Básico, editora, Kanni Wignaraja.http://www.undp.org/content/dam/undp/library/capacitydevelopment/spanish/Capacity_Development_A_UNDP_Primer_Spanish.pdf

REAL ACADEMIA ESPAÑOLA. Diccionario de la lengua española, 23. a ed., [versión 23.4 en línea]. <https://dle.rae.es> [Tomado el 15 de noviembre de 2020].

Sánchez, L., Pino, E. Espejo, M. (2008). "Una mirada a la participación comunitaria en el proceso de contraloría social. República Bolivariana de Venezuela". (https://www.researchgate.net/publication/262629095_Una_mirada_a_la_participacion_comunita ria_en_el_proceso_de_contraloria_social). 
Secretaría Nacional de Descentralización. (2016). Boletín digital Panamá Avanza. (http://www.descentralizacion.gob.pa/tmp/file/45/boletininformativosnd.pdf).

\section{Leyes}

Ley Nº 27901-A. Gaceta Oficial de la República de Panamá, Ciudad de Panamá, 29 de octubre de 2015 .

https://www.gacetaoficial.gob.pa/pdfTemp/27901_A/GacetaNo_27901a_20151030.pdf

Ley N 17210. Gaceta Oficial de la República de Panamá, Ciudad de Panamá, 24 de octubre de 1972.

https://www.asamblea.gob.pa/APPS/LEGISPAN/PDF_NORMAS/1970/1972/1972_028_ 2256.pdf

Ley N 25176. Gaceta Oficial de la República de Panamá, Ciudad de Panamá, 15 de noviembre de 1972. 